\title{
Real-time irradiation system using patterned light to actuate light-driven on-chip gel actuators
}

\author{
Yuha Koike ${ }^{1 *} \mathbb{D}$, Shunnosuke Kodera' ${ }^{1}$, Yoshiyuki Yokoyama² and Takeshi Hayakawa' ${ }^{1}$
}

\begin{abstract}
A light-driven gel actuator is a potential candidate for a single-cell manipulation tool because it allows cells to be manipulated while ensuring less damage. Moreover, a large number of actuators can be integrated into a microfluidic chip because no wiring is required. Previously, we proposed a method for cell manipulation using light-driven gel actuators. However, the system used in the previous work did not allow the targeted cells to be manipulated in real time because the system used in the previous work could only irradiate preprogrammed patterned light. Moreover, when a large number of gel actuators are integrated into a chip, the Gaussian distribution of the laser light source results in the response characteristics of the gel actuators varying with the location of the actuator. In this work, we constructed a system that homogenized the intensity of the patterned light used for irradiation, allowing multiple gel actuators to be driven in parallel in real time. The intensity-homogenized patterned light improved the variations in the response characteristics of the gel actuators, and as a result, we succeeded in actuating gel actuators with various light patterns in real time.
\end{abstract}

Keywords: Micromanipulation, Cell manipulation, On-chip manipulation, Gel actuator, Digital mirror device (DMD)

\section{Introduction}

Micromanipulation techniques are essential for analyzing cells or tissues in the fields of medicine and cell biology [1]. Mechanical micromanipulation is a standard method for cell manipulation and analysis [2]. This method uses a mechanical micromanipulator having multiple degrees of freedom (DOF) and gives high positioning accuracy. Mechanical micromanipulators can be equipped with various end effectors, such as glass capillaries, nanoneedles, or electrode sensors [3], and can thus be used for a range of cell manipulations, including fixing, rotating and injecting DNA or RNA into cells. Consequently, they are widely used for medical and biological research. However, mechanical micromanipulators require highly skilled operators, leading to low throughput and fewer

\footnotetext{
*Correspondence: yuha-o-moody@mnrobo.mech.chuo-u.ac.jp 1 Department of Precision Mechanics, Chuo University, Tokyo, Japan Full list of author information is available at the end of the article
}

repeatable processes. Another potential method of cell manipulation is on-chip cell manipulation performed in a microfluidic device. A microfluidic device consists of microchannels or microchambers and may integrate sensors and actuators. In the microchannel or microchamber, the DOF of the target cells is confined to one or two degrees, reducing fluctuations in the target cell's position and enabling high throughput and high repeatability for cell manipulations on a chip. On-chip cell manipulations use noncontact methods or contact methods. Noncontact methods use external forces, such as electric [4], magnetic [5], acoustic [6], or optical [7] forces to manipulate the target cells without contact. However, these manipulating forces are generally weak and in the order of piconewtons to nanonewtons, thus limiting noncontact methods for cell manipulation.

By contrast, contact methods that use microstructures, such as microactuators or microrobots that are driven by optical [8], magnetic $[9,10]$, or electric [11] forces, make

\section{Springer Open}

(C) The Author(s) 2022. Open Access This article is licensed under a Creative Commons Attribution 4.0 International License, which permits use, sharing, adaptation, distribution and reproduction in any medium or format, as long as you give appropriate credit to the original author(s) and the source, provide a link to the Creative Commons licence, and indicate if changes were made. The images or other third party material in this article are included in the article's Creative Commons licence, unless indicated otherwise in a credit line to the material. If material is not included in the article's Creative Commons licence and your intended use is not permitted by statutory regulation or exceeds the permitted use, you will need to obtain permission directly from the copyright holder. To view a copy of this licence, visit http://creativecommons.org/licenses/by/4.0/. 
direct contact with the target cells, similar to mechanical micromanipulators. These actuators and robots can manipulate with high positioning accuracy and have stronger manipulation forces than noncontact methods. Thus, contact methods are used for applications requiring a large force or high-precision, such as the analysis of cellular responses to mechanical stimulation [12]. However, contact methods incur the risk of cell damage when the microstructures contact the target cells. To overcome these problems, on-chip gel actuators that have a similar softness to cells are attracting considerable attention [13-19]. Although they use a contact method, on-chip gel actuators cause less physical damage to cells because they are soft. On-chip gel actuators are commonly made of poly(N-isopropylacrylamide) (PNIPAAm), a thermoresponsive hydrogel [20]. PNIPAAm swells in water when its temperature is less than the lower critical solution temperature (LCST) $\left(\leq 32^{\circ} \mathrm{C}\right)$ and shrinks when its temperature is higher than the LCST $\left(\geq 32^{\circ} \mathrm{C}\right)$. Thus, by controlling the temperature of the PNIPAAm, we can use this volume change as an actuator. Previously, we proposed a method for driving an on-chip gel actuator made of PNIPAAm using light irradiation [18, 19]. In this method, gel actuators are fabricated on a glass substrate with a light-absorbing pattern. On irradiating the light absorber with light, the temperature of the light absorber increases and the gel actuators shrink, as shown in Fig. 1a. In our previous work $[18,19]$, we succeeded in integrating a large number of gel actuators into a microfluidic chip and driving multiple actuators by irradiating the integrated gel actuators with patterned light. Additionally, we used the integrated gel actuators to demonstrate various cell manipulations, including cell transporting, trapping and sorting. However, it was difficult to manipulate the targeted cells with arbitrary timings because the previous system could only irradiate the gel with preprogrammed light patterns. Moreover, the irradiated light had an intensity distribution of Gaussian distribution in a workspace of the manipulation. Therefore, when numerous gel actuators were integrated into a microfluidic chip, shrink responses of the actuators were different and it was difficult to control actuation timings
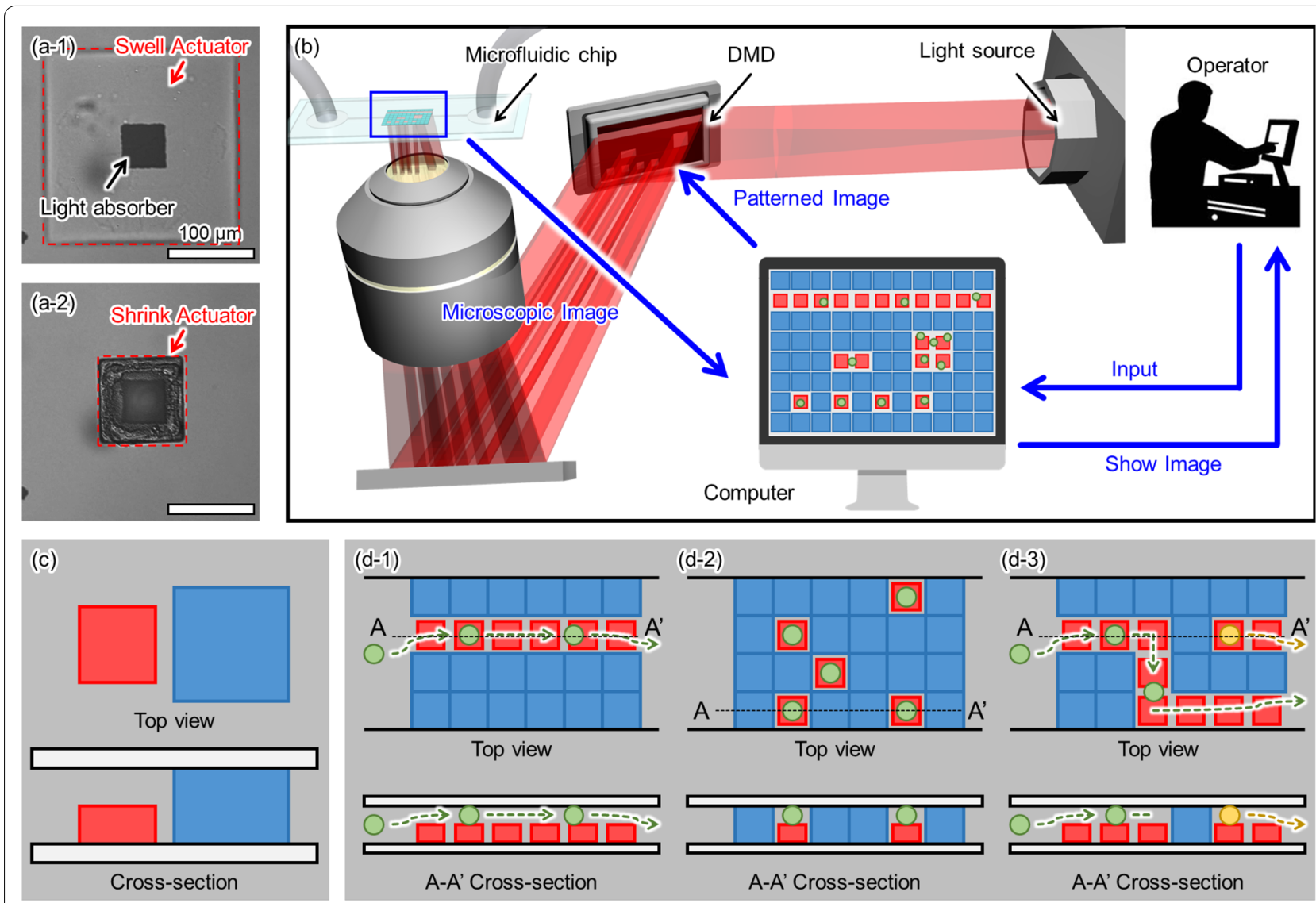

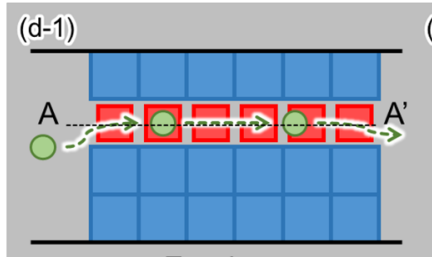

Top view



A-A' Cross-section

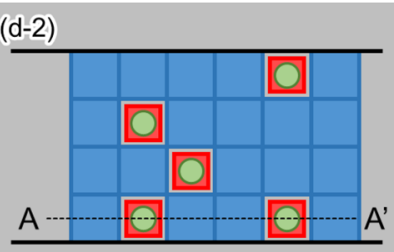

Top view

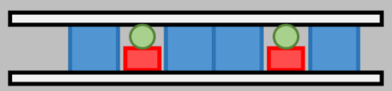

A-A' Cross-section



Top view

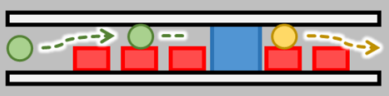

A-A' Cross-section

Substrate

: Swell actuator

Shrink actuator

Fig. 1 Concept of the proposed on-chip gel actuator. a Light drive for the gel actuator. $\mathbf{b}$ Concept of the irradiation system of patterned light using a digital micromirror device. $\mathbf{c}$ Displacement of light-driven gel actuator in integrated gel actuator. $\mathbf{d}$ Example of cell manipulations 
of actuators. In this paper, we describe the construction of a system for generating light patterns in real time and homogenizing the intensity of the light. We evaluate the switching responses of the irradiated light and the homogeneity of the patterned light. Finally, we confirm that patterned gel actuators can be actuated with approximately the same response times and actuated with various light patterns in real time.

\section{Concept}

Figure $1 \mathrm{~b}$ presents the conceptual diagram of a system that uses a digital micromirror device (DMD) to drive multiple gel actuators. First, the operator observes a microscopic image on a control PC. Next, the operator inputs coordinates or areas on the image using a mouse and keyboard to determine the light irradiation point or area. Finally, the computer generates a patterned image based on the input coordinates or area data and sends it to the DMD to generate irradiation with the patterned light. This process runs at $30 \mathrm{fps}$ in real time, and the operator can switch the points or areas of light being irradiated in real time. Thus, by using proposed system and integrated gel actuators, we can perform cell manipulations such as cell transport (Fig. 1b-1), cell trapping (Fig. 1b-2) or cell sorting (Fig. 1b-3) in microfluidic devices.

\section{Experiments}

\section{Optical system for irradiating with patterned light}

The optical system we designed using a DMD to irradiate with patterned light is shown in Fig. 2a-1. An infrared (IR) laser (SP-020P-AHS-S, SPI Lasers UK Ltd., Hedge End, UK; wavelength $=1064 \mathrm{~nm}$; maximum power $=20$ W) was used as the light source to drive the gel actuators. An Iris diaphragm (Iris) was used to reshape the laser beam diameter, and two lenses, $L 1$ and $L 2$, were used to expand the laser beam to match the size of the DMD. DMDs are commonly used to irradiate with patterned light; for example, in maskless exposure equipment or external devices for microscopes [21, 22]. The DMD we used to generate patterned light (LC4500-RGB-EKT, Keynote Photonics, Texas, United States) had a resolution of 912 x 1140 pixels. The patterned light generated by the DMD irradiated the microfluidic device through three lenses: L3, L4 and an objective lens (LMPlan $\mathrm{N}$ 10x/0.30 Na IR, Olympus, Tokyo, Japan). A dichroic mirror (86-694, Edmund Optics, Barrington, USA; cutoff wavelength $=950 \mathrm{~nm}$ ) was used to reflect the IR laser beam to observe the device with visible light.

Microscopic images were acquired using a complementary metal oxide semiconductor (CMOS) camera (GS3U3-23S6C-C, FLIR Systems Japan K.K., Tokyo, Japan). Usually, when visible light is focused on the focal plane

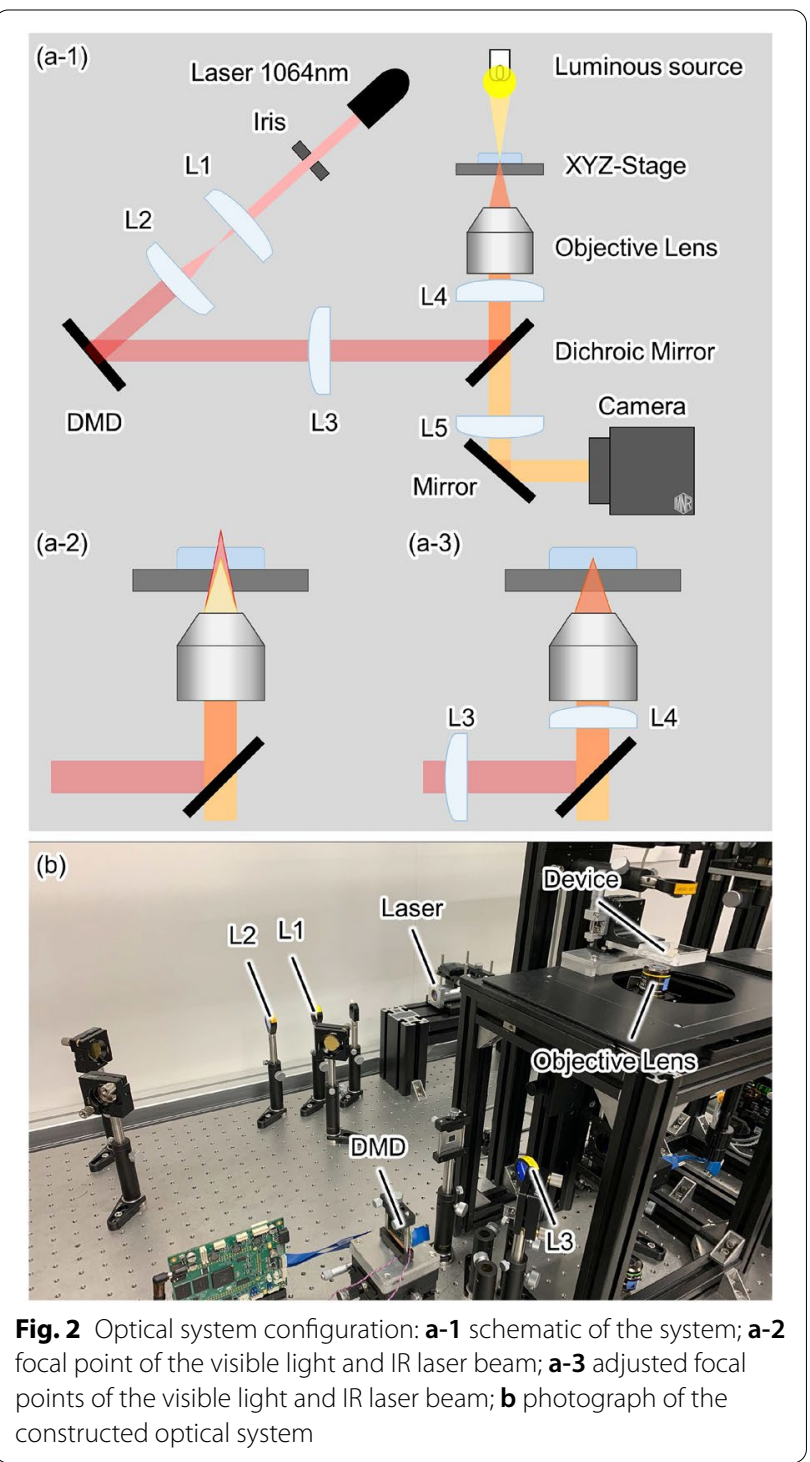

of an objective lens, the IR laser beam is out of focus as a result of chromatic aberrations at different wavelengths, as shown in Fig. 2a-2. To counter this, we used lenses $L 3$ and L4 to focus both the visible light and the IR laser beam onto the same focal plane (Fig. 2a-3). When L3 was moved closer to the DMD, the focal point of the IR laser beam moved to the bottom side of the microfluidic device, and when $L 3$ was moved away from the DMD, the focal point of the IR laser beam moved to the upper side of the microfluidic device. Figure $2 b$ shows a photograph of the optical system we constructed.

\section{Software configuration}

We constructed software that generates arbitrary images from inputs by an operator and sends them to the DMD 


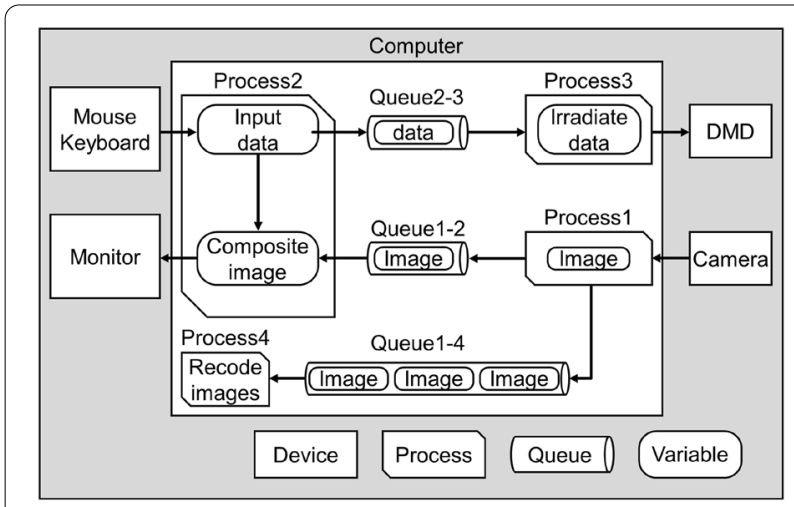

Fig. 3 Schematic of the software

to generate patterned light in real time. Figure 3 shows a schematic of the software. The system has four processes that work independently in parallel. Additionally, we used three queues between each process to exchange data; e.g., microscopic images and coordinates from the mouse and/or keyboard. A queue is commonly used in programming processes when information must be exchanged safely among multiple processes. Process 1 acquires a microscopic image from the camera and transfers the image to Queue 1-2 if the queue is empty. When we record a video, Process 1 transfers the acquired image to Queue 1-4. Process 2 receives the image from Queue 1-2 and displays it on the monitor for the operator. Then, the operator inputs the coordinates for the image for light patterns using the mouse or keyboard. Process 2 receives the input data, which is then used to draw the irradiated points on the displayed image. Simultaneously, the input data are transferred to Queue 2-3. Process 3 receives the input data from Queue 2-3 and uses these data to generate an image for the light patterns. The generated image is subsequently displayed on the DMD to irradiate the surface with patterned light. Process 4 functions when we record a video; it receives images from Queue 1-4 and makes a video file. We used a computer with an Intel core i7-8700 processor (6 physical cores, $3.20 \mathrm{GHz}$ ), $64 \mathrm{~GB}$ of DDR4 memory and the Windows 10 operating system. The software was developed using Python 3.6.8 .

\section{Homogenization of the light intensity}

Next, we use the DMD to homogenize the intensity of the patterned light. Each mirror of the DMD can be independently driven and switched on/off. Thus, light reflected from the DMD can display a light pattern with an arbitrary image, as shown in Fig. 4a-1. Also, we can change the intensity of the light reflected from the DMD by changing the switching frequency of the individual mirrors in the DMD. Inputting a grayscale image to the



Fig. 4 a Photographs of the surface of the DMD. $\mathbf{b}$ Homogenization process of a light intensity

DMD allow us to generate the intensity gradient shown in Fig. 4a-2. Basically, we use binary images to generate light pattern in this study. However, the intensity distribution of the IR laser beam used to drive the gel actuators was Gaussian and precise intensity distribution of a laser reflected by the DMD is unknown. Thus, we evaluate the distribution of the reflected laser and use grayscale images to homogenize an intensity of reflected laser.

First, we evaluate intensity distribution of reflected laser by inputting an image with the highest brightness values in all pixels to the DMD, in other words, inputting a white image. By inputting the white image to the DMD and measuring an intensity distribution of the reflected light by using a laser profiler, we can get actual intensity distribution of the reflected laser. In this system, it is difficult to increase intensities at pixels with low values. Thus, we realize homogenization of intensities of the reflected laser by suppressing intensities of pixels with high values by inputting gray values to the DMD. To measure the light intensity of the patterned light, we replace the camera shown in Fig. 2 a with a laser profiler (LBP2-HR-VIS3, Japan Laser Corporation, Tokyo, Japan). Detailed procedure of the homogenization are explained as follows and shown in Fig. 4b.

- Input a white image to the DMD.

- Measure the intensity distribution of a light reflected from the DMD $\left(I_{x, y}\right)$ by using the laser profiler.

- Use the following Eq. (1) to determine a reflection rate of each pixel of the DMD.

$$
r_{x, y}=I_{\min } / I_{x, y}
$$


Here, $r_{x, y}$ is reflection rate of an input image at pixel $(x, y)$ and $I_{\min }$ is the minimum value of the measured intensity distribution among all pixels. Because $I_{x, y}$ means intensity distribution of the reflected laser, the values $I_{\text {min }} / I_{x, y}$ give reflection rate for each pixel to suppress intensities to $I_{\text {min }}$ for all pixels.

- Finally, we can acquire homogenized reflected laser with minimum intensity values by inputting intensity values multiplied by $r_{x, y}$ to the DMD.

\section{Fabrication}

Microfabrication processes were used to fabricate the on-chip gel actuators and light absorbers. The detailed fabrication process is explained in [18]. To pattern the PNIPAAm, we used photo-processable PNIPAAm (Bioresist $^{\oplus}$, Nissan Chemical Corporation, Tokyo, Japan).

\section{Results and discussion}

\section{Calibration and evaluation of the position of the patterned} light

First, we calibrated the position irradiated with the patterned light on a microscopic image. We irradiated three corners of a box-shaped irradiation area. The light was used to irradiate a glass substrate with a $\mathrm{Cr}$ layer and we acquired the coordinates of the reflected light on a microscopic image, as shown in Fig. 5a. The three acquired positions were saved and the coordinates of the DMD and microscopic camera were calibrated. Next, we irradiated the substrate with the block check pattern (Fig. 5b) to confirm that the calibration was correct. Then we adjusted the chromatic aberration of the IR laser beam and the visible light. In the optical system we constructed, the same objective lens was used to irradiate

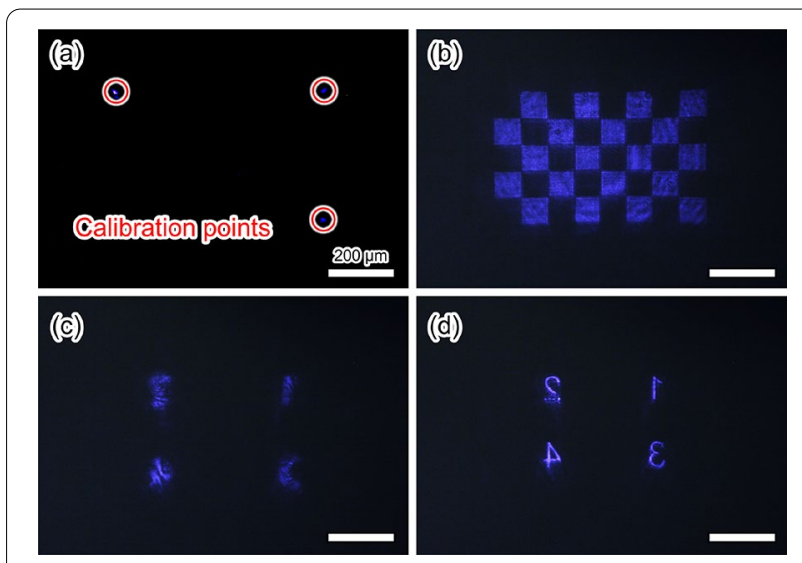

Fig. 5 Microscopic images of irradiation with patterned light: a calibration of irradiated position, $\mathbf{b}$ block check pattern, $\mathbf{c}$ before focusing, $\mathbf{d}$ after focusing and observe the patterned light. Thus, the IR laser beam was unfocused and the patterned light was blurred when we focused the device with visible light, as shown in Fig. 5c. To correct this, we adjusted the position of lens L3 until the IR laser beam had the same focal plane as the visible light. Adjusting the chromatic aberration and focusing the device in this way allowed the patterned light to irradiate on the focal plane without blurring, as shown in Fig. 5d. Finally, we used the following process to evaluate the positioning accuracy of the irradiated points.

- Irradiate the square shape with a side length of 30 $\mu \mathrm{m}$ to four corners and center of the irradiation area (Fig. 6a) and capture the image.

- Process the binarization of the captured image (Fig. 6b).

- Calculate the center of each irradiation area.

- Compare the acquired center of the area and the input values.

The maximum difference in positions of the irradiated point was approximately $3.5 \mu \mathrm{m}$, and the average difference was $2.1 \mu \mathrm{m}$. These values are thought to be small enough to manipulate biological cells of a size of approximately $10-20 \mu \mathrm{m}$.

\section{Resolution of the generated light pattern}

First, we confirmed the minimum size of the light patterns. We irradiated a glass surface coated with a $\mathrm{Cr}$ layer with patterned light and used the CMOS camera to observe the reflected patterns. When we irradiated the surface with a square pattern smaller than 4 pixels $x 4$ pixels from DMD, we were not able to observe any reflected light. Thus, we concluded that 4 pixels $x 4$ pixels is the smallest observable irradiation light pattern, corresponding to an actual light pattern on the focal plane of approximate size $2 \mu \mathrm{m} \times 2 \mu \mathrm{m}$. Next, we evaluate the spatial resolution of the generated light pattern. To determine the resolution, which we took as the minimum distance between two light patterns with distinguishable borders, we irradiated the glass surface with two square



Fig. 6 Image used to evaluate the positioning accuracy: a row image; $\mathbf{b}$ after binarization 
light patterns of 20 pixels $\times 20$ pixels at various distances apart, as shown in Fig. 7. The square light pattern of 20 pixels x 20 pixels shown in Fig. 7a corresponds approximately to an actual size of $12 \mu \mathrm{m} \times 12 \mu \mathrm{m}$. The photos on the left of Fig. $7 \mathrm{a}-\mathrm{c}$ were taken with the CMOS camera and intensity plots of the images are shown on the right. Figure $7 \mathrm{a}$ shows that the irradiated light has a Gaussian
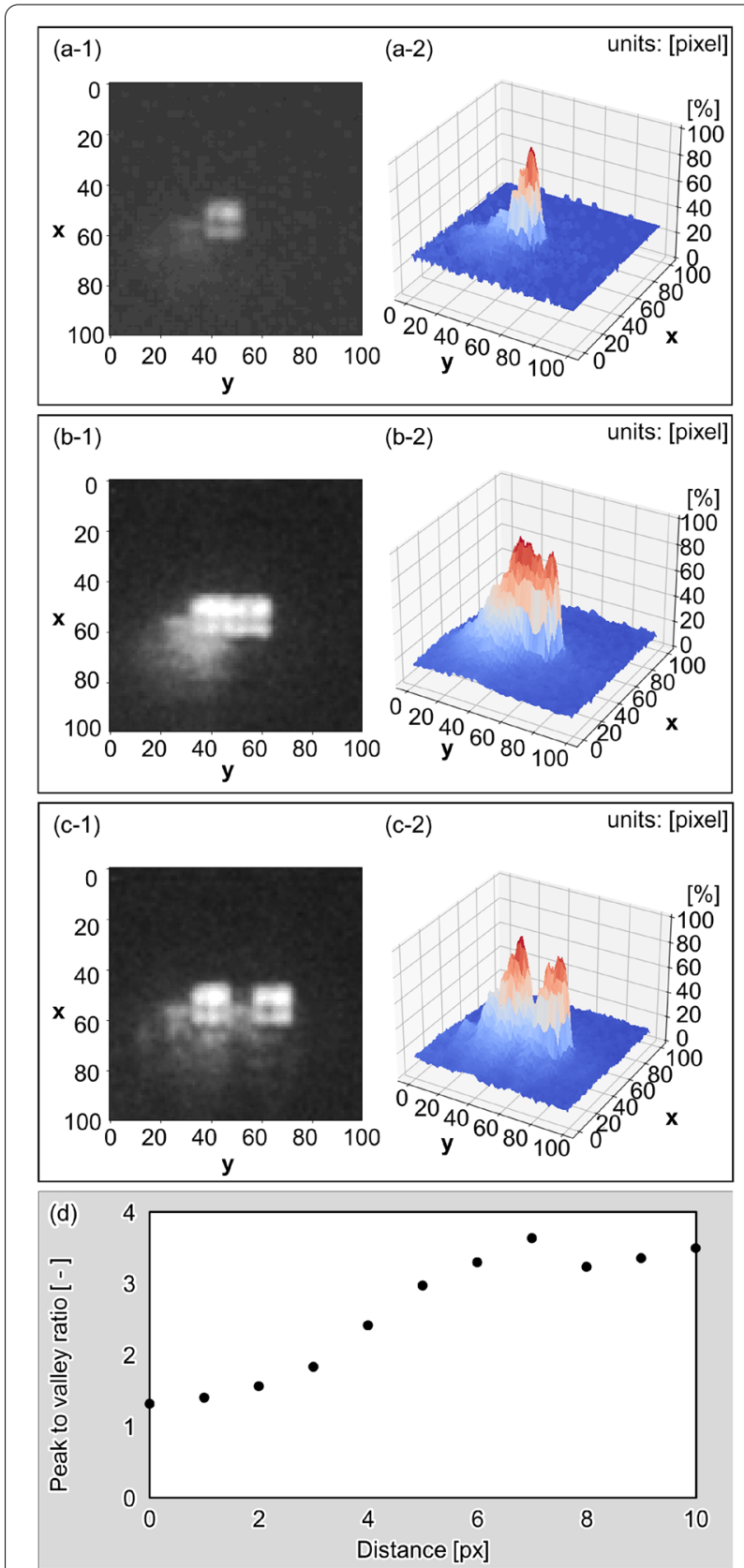

Fig. 7 Evaluation of the resolution of the light patterns. Irradiation with a square pattern of 20 pixels $\times 20$ pixels a single pattern; $\mathbf{b}$ two patterns spaced at 0 pixels; $\mathbf{c}$ two patterns spaced at 7 pixels; $\mathbf{d}$ plot of the resolution of the generated light pattern intensity distribution. Additionally, the reflected laser has a small distribution near the left bottom corner of the image. This small distribution is thought to be unexpected diffracted light. However, the intensity of this distribution is small compared to the main distribution of the pattern, thus it can be ignored. Then, we irradiate two square patterns with various distances, as shown in Fig. 7b-d. We evaluated the distinguishable border with a peak to valley ratio defined by the following equation.

$$
\text { peak to valley ratio : } \frac{\left(p_{1}+p_{2}\right) / 2}{v}
$$

Here, $p_{1}$ is the highest intensity of the left square and $p_{2}$ is the highest intensity of the right square. The value of $v$ is the lowest intensity between left and right squares. When the distance between the two squares was 0 pixels, the peak valley ratio was 1.3 , as shown in Fig. $7 \mathrm{~b}$ and $\mathrm{d}$. When the distance between the two squares was 7 pixels, the peak to valley ratio became 3.6, as shown in Fig. 7c and $d$. The value of the peak to valley ratio saturated at around 3.3-3.6, as shown in Fig. 7d. From this, we determined the resolution of the patterned light to be approximately 7 pixels, which corresponds to an actual distance of $4 \mu \mathrm{m}$ on the focal plane of the microscope. This value is thought to be small enough to manipulate biological cells of a size of approximately $10-20 \mu \mathrm{m}$.

\section{Evaluation of the switching responses of the light irradiation}

This subsection describes how we confirmed that the DMD was switching correctly. We evaluated the processing time when sending an image to the DMD for the following processes.

- Send an image to the DMD $N$ times with various frequencies

- Measure the sending time and define it as $T$.

- At each frequency, count the number of trials if $T$ is within the cycle time and define the number as $N_{S}$.

- Calculate the success rate $A=N_{S} / N$.

Figure 8 shows a plot of the success rate $A$ for various switching frequencies. Below $30 \mathrm{~Hz}$, the irradiation was switched completely within the cycle time. However, the success rate decreased to $98 \%, 95 \%$ and $85 \%$ at $40 \mathrm{~Hz}, 50$ $\mathrm{Hz}$ and $60 \mathrm{~Hz}$, respectively. From the success rate, the practical response frequency of the system was estimated to be approximately $30 \mathrm{~Hz}$.

\section{Homogenization of the light intensity}

To confirm that the IR laser beam was homogeneous, we evaluated its intensity using a laser profiler. First, we used the laser profiler to measure the intensity values $\left(I_{x, y}\right)$ of 
every pixel of the light reflected by the DMD. Then, we used Eq. (1) to calculate the input values $\left(i_{x, y}\right)$ at each pixel for the images input to the DMD. We then measured the homogenized intensity values for every pixel again. Figure 9a shows the intensity distribution before homogenization. The intensity of the upper left side of the image was high and the intensity of the lower right side was low. After homogenization, the intensity of the upper left side was suppressed and the overall intensity was almost completely homogenized, as shown in Fig. 9b. The maximum and minimum values and coefficient of variation $(\mathrm{CV})$ of the intensity distribution before and after homogenization are listed in Table 1. Before homogenization, the maximum and minimum values of the intensity were 3251 and 980, respectively, in 4096 steps and the CV was 0.153. After homogenization, the maximum value of the intensity decreased to 1509 in 4096 steps and the CV was 0.066 . Thus, the homogeneity of the light intensity was improved when corrected with Eq. (1).

Although this method succeeded in homogenizing the laser intensity, the maximum value of the intensity decreased. This decrease in intensity is a disadvantage when we need a high intensity, and so we revise the equation for homogenization to adjust the maximum intensity and homogeneity of the light intensity, and we defined the homogenization rate $R_{h}$ using the following equations.

$$
\begin{aligned}
& i_{x, y}^{\prime}=\left\{\begin{array}{lll}
I_{\min } / I_{x, y} & \text { if } & I_{x . y} \leq i_{h} \\
i_{h} / I_{x, y} & \text { if } & I_{x . y}>i_{h}
\end{array}\right)
\end{aligned}
$$

Here, $i_{x, y}^{\prime}$ is the input value for pixel $x, y$, And $I_{\max }$ and $I_{\text {min }}$ are the measured maximum and minimum intensities of the laser before homogenization. $i_{h}$ is the target maximum intensity after homogenization, which can be set between $I_{\text {min }}$ and $I_{\max }$. For example, when the value of $i_{h}$ is set to $I_{\max }$, the laser is not homogenized and the homogenization rate is $0 \%$. Similarly, when the value of $i_{h}$ is set to $I_{\text {min }}$, Eq. (3) and Eq. (1) become the same. Then,

Table 1 Comparison of intensity values before and after homogenization

\begin{tabular}{lll}
\hline & Before & After \\
\hline CV & 0.153 & 0.066 \\
Max. & 3251 & 1509 \\
Min. & 980 & 809 \\
\hline
\end{tabular}

the laser is homogenized and the homogenization rate is $100 \%$. Therefore, we can use Eq. (3) and Eq. (4) to adjust the homogenization rate or required maximum intensity $i_{h}$. The maximum and the minimum intensities and $\mathrm{CV}$ after homogenization with Eq. (3) for various homogenization rates $R_{h}$ are summarized in Table 2 .

\section{Using the constructed system to drive gel actuators}

We used the system we constructed to drive the gel actuators and compared the results with the previous system. We fabricated $25 \times 25=625$ actuators on a glass substrate, as shown in Fig. 10. These actuators were square with sides of length $50 \mu \mathrm{m}$. The gap between each gel

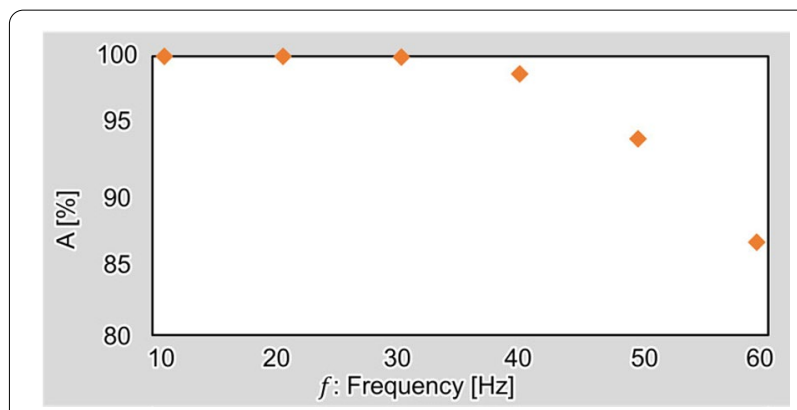

Fig. 8 Evaluation of the switching response

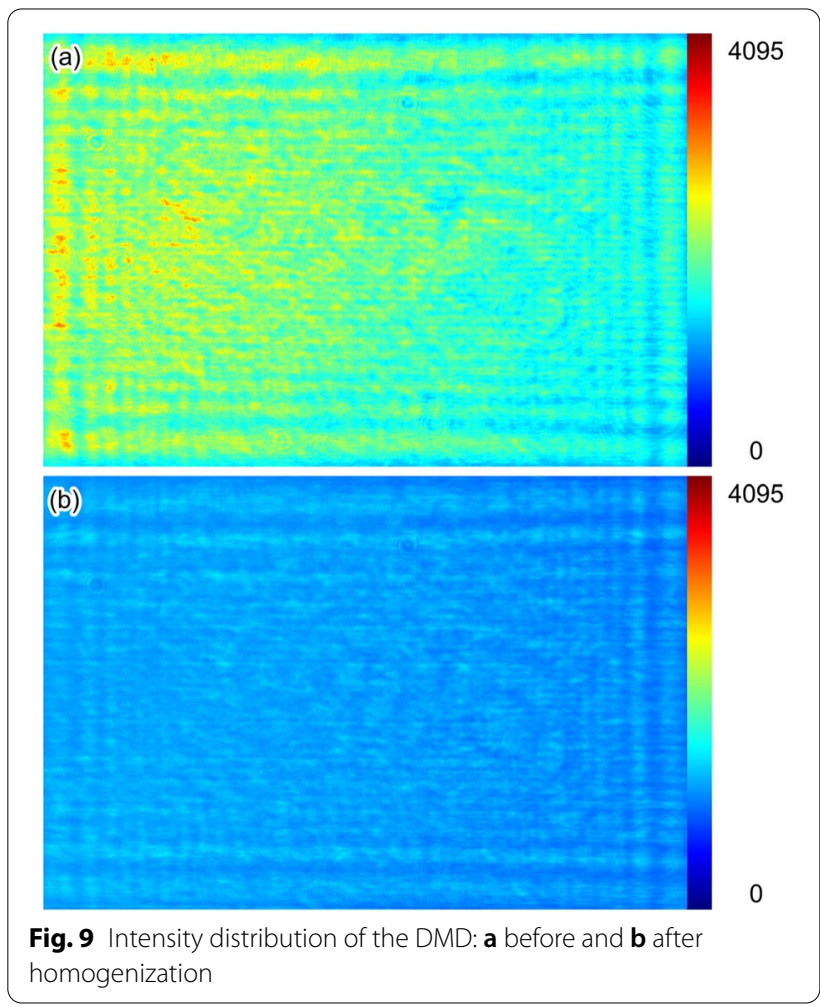


Table 2 Comparison of intensity values before and after homogenization for various homogenization rates $R_{h}$

\begin{tabular}{lllllll}
\hline $\boldsymbol{R}_{\boldsymbol{h}}$ & $\mathbf{0 \%}$ & $\mathbf{2 0 \%}$ & $\mathbf{4 0 \%}$ & $\mathbf{6 0 \%}$ & $\mathbf{8 0 \%}$ & $\mathbf{1 0 0 \%}$ \\
\hline C. V. & 0.153 & 0.149 & 0.146 & 0.100 & 0.067 & 0.066 \\
Max. & 3251 & 3080 & 3116 & 2383 & 1497 & 1509 \\
Min. & 980 & 995 & 981 & 947 & 821 & 809 \\
\hline
\end{tabular}



Fig. 10 Using the DMD to drive gel actuators: a previous system without homogenization, $\mathbf{b}$ constructed system with homogenization

actuator in the shrunken state was $20 \mu \mathrm{m}$. When the actuators were in water, the gaps were filled by the swelling of the gel, and the side length became $70 \mu \mathrm{m}$ when the gel was swollen. The area irradiated with patterned light was approximately $630 \mu \mathrm{m}$ wide $\mathrm{x} 420 \mu \mathrm{m}$ high. This meant that with the constructed system we were able to drive $9 \times 6=54$ gel actuators in parallel. We irradiated five points on the integrated gel actuators in parallel and evaluated the difference in their shrink responses. Using the previous system with nonhomogenized light, the gel actuators at Positions 1 and 3 were shrunk and the other gel actuators were swollen approximately $0.2 \mathrm{~s}$ after irradiation, as shown in Fig. 10a. However, using the constructed system with homogenized light with a $50 \%$

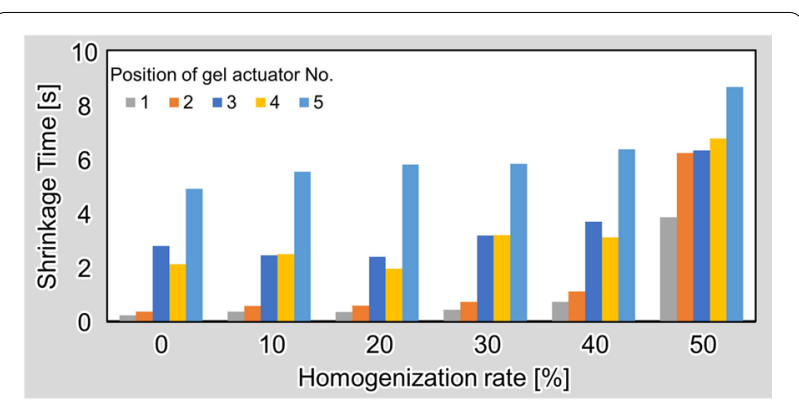

Fig. 11 Shrinkage time of a gel actuator for various homogenization rates

homogenization rate, the gel actuators at all positions were successfully shrunk approximately $5.9 \mathrm{~s}$ after irradiation, as shown in Fig. 10b. This confirmed that homogenization using Eq. (3) works well. Next, we measured the shrinkage time of the gel actuators at every position. Figure 11 is a plot of the shrinkage time of the gel actuators for various homogenization rates. The experiment was conducted with a homogenization rate of $50 \%$ or less. These results highlight the large difference between the shrinkage times of the individual actuators before homogenization. After homogenization, the shrinkage times of actuators 1,2 , and 4 were almost the same. The $\mathrm{CVs}$ of the shrinkage times at each homogenization rate are listed in Table 3. For a $0 \%$ homogenization rate, the $\mathrm{CV}$ was 0.83 . For a $50 \%$ homogenization rate, the $\mathrm{CV}$ was 0.24 . This confirmed that homogenizing the laser beam reduces the variation in the shrinkage times of the gel actuators.

Finally, we demonstrated using various light patterns to drive gel actuators. Figure 12 shows microscopic images of the gel actuators driven in parallel. For the integrated gel actuators, only actuators irradiated with the patterned light were shrunk. A supplementary video of this demonstration is available online.

\section{Conclusions}

In this work, we constructed an irradiation system of patterned light for parallel actuation of multiple gel actuators on a microfluidic chip. We constructed an optical system to irradiate gel actuators with patterned light using 
Table 3 Comparison of shrinkage times for different homogenization rates

\begin{tabular}{lllllll}
\hline \multicolumn{7}{c}{ Homogenization rate $\boldsymbol{R}_{\boldsymbol{h}}$} \\
\cline { 2 - 7 } & $\mathbf{0} \%$ & $\mathbf{1 0 \%}$ & $\mathbf{2 0 \%}$ & $\mathbf{3 0 \%}$ & $\mathbf{4 0 \%}$ & $\mathbf{5 0 \%}$ \\
\hline C.V. & 0.83 & 0.82 & 0.88 & 0.73 & 0.67 & 0.24 \\
\hline
\end{tabular}
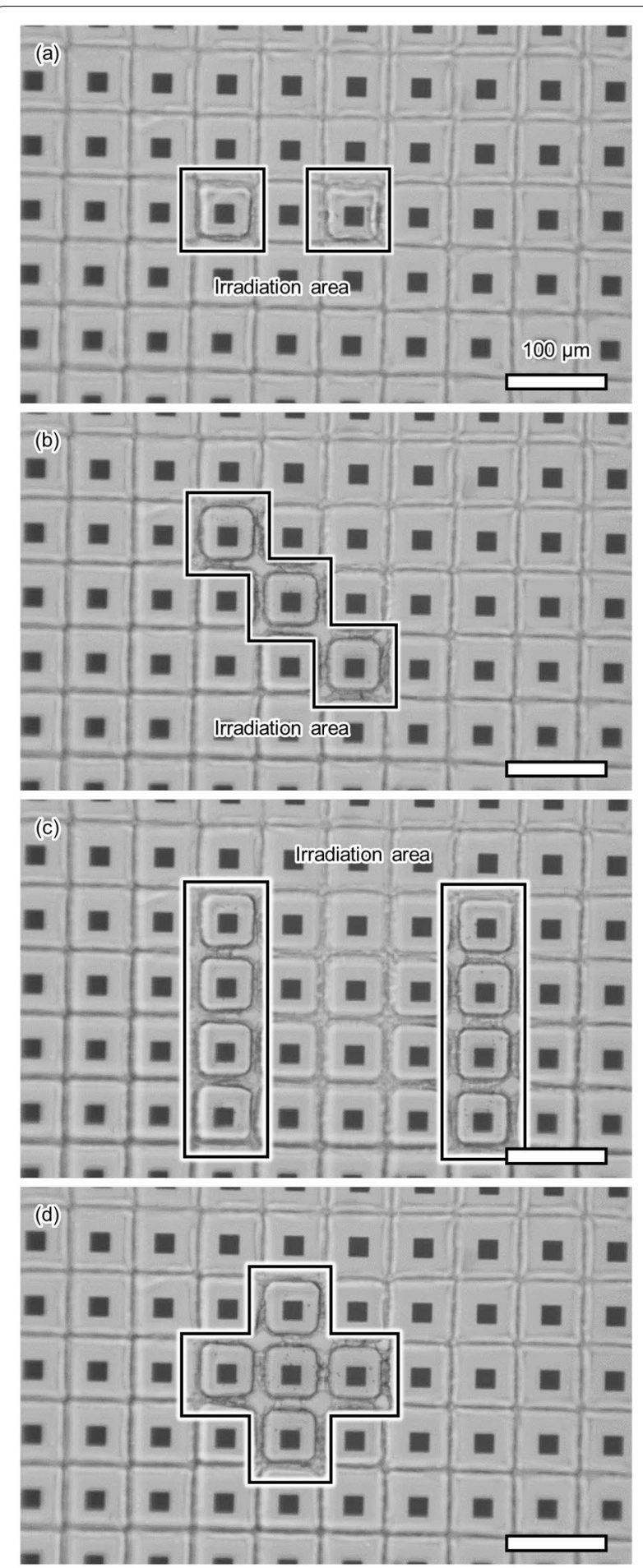

Fig. 12 Driving gel actuators with various light patterns. a two-point drive; $\mathbf{b}$ three-point drive; $\mathbf{c}$ two-line drive; $\mathbf{d}$ cross-shaped drive a DMD and software to homogenize the IR laser beam intensity. The constructed system functioned successfully up to $30 \mathrm{~Hz}$. We evaluated the CV of the intensity distribution before and after homogenization. Homogenizing the intensity improved the CV from 0.153 to 0.066 . We confirmed the drive characteristics of the gel actuators before and after homogenization. Before homogenization, the shrink responses of the gel actuators varied widely. However, homogenization succeeded in reducing the variation in intensities. Finally, we confirmed that we could use patterned light to drive gel actuators, and were successful in driving multiple actuators and switching them on/off in real time. Future work includes developing our constructed system to implement various cell manipulations in parallel.

\section{Acknowledgements}

Bioresist was provided by Nissan Chemical Corporation. We thank Michael Luetchford, BTech, from Edanz Group (https://en-author-services.edanzgroup. $\mathrm{com} /$ ) for editing a draft of this manuscript.

\section{Authors' contributions}

YK, SK, and TH contributed equally to this work. YK, SK, and TH were responsible for the conception and design of the study, collection of data, analysis and interpretation of data, drafting of the manuscript and critical revision of the manuscript for important intellectual content. YY was responsible for the design of the study and critical revision of the article for important intellectual content. All authors read and approved the final manuscript.

\section{Funding}

This research was supported by a Chuo University Personal Research Grant, Grant for Special Research, and JSPS KAKENHI Grant Numbers JP 21J14498 and 21K18703.

\section{Availability of data and materials}

Not applicable.

\section{Declarations}

\section{Competing interests}

The authors declare that they have no competing interests.

\section{Author details}

${ }^{1}$ Department of Precision Mechanics, Chuo University, Tokyo, Japan. ${ }^{2}$ Toyama Industrial Technology Research and Development Center, Toyama, Japan.

Received: 21 October 2021 Accepted: 4 February 2022

Published online: 19 February 2022

\section{References}

1. Lindström S, Andersson-Svahn H (2010) Overview of single-cell analyses: microdevices and applications. Lab Chip 10(24):3363-3372 
2. Yanagida K, Katayose H, Yazawa H, Kimura Y, Konnai K, Sato A (1999) The usefulness of a piezo-micromanipulator in intracytoplasmic sperm injection in humans. Hum Reprod 14(2):448-453

3. Obataya I, Nakamura C, Han S, Nakamura N, Miyake J (2005) Nanoscale operation of a living cell using an atomic force microscope with a nanoneedle. Nano Lett 5(1):27-30

4. Wu C, Chen R, Liu Y, Yu Z, Jiang Y, Cheng X (2017) A planar dielectrophoresis-based chip for high-throughput cell pairing. Lab Chip 17(23):4008-4014

5. Shields CW IV, Wang JL, Ohiri KA, Essoyan ED, Yellen BB, Armstrong AJ, López GP (2016) Magnetic separation of acoustically focused cancer cells from blood for magnetographic templating and analysis. Lab Chip 16(19):3833-3844

6. Guo F, Mao Z, Chen Y, Xie Z, Lata JP, Li P, Ren L, Liu J, Yang J, Dao M et al (2016) Three-dimensional manipulation of single cells using surface acoustic waves. Proc Natl Acad Sci 113(6):1522-1527

7. Ashkin A, Dziedzic JM, Yamane T (1987) Optical trapping and manipulation of single cells using infrared laser beams. Nature 330(6150):769-77

8. Onda K, Arai F (2012) Multi-beam bilateral teleoperation of holographic optical tweezers. Opt Express 20(4):3633-3641

9. Sakuma S, Yamanishi Y, Arai F (2009) Magnetically driven microtools actuated by a focused magnetic field for separating of microparticles. J Robot Mechatron 21(2):209

10. Hagiwara M, Kawahara T, Yamanishi Y, Masuda T, Feng L, Arai F (2011) Onchip magnetically actuated robot with ultrasonic vibration for single cell manipulations. Lab Chip 11(12):2049-2054

11. Ito K, Sakuma S, Kimura M, Takebe T, Kaneko M, Arai F (2016) Temporal transition of mechanical characteristics of huvec/msc spheroids using a microfluidic chip with force sensor probes. Micromachines 7(12):221

12. Park K, Mehrnezhad A, Corbin EA, Bashir R (2015) Optomechanical measurement of the stiffness of single adherent cells. Lab Chip 15(17):3460-3464

13. Ichikawa A, Arai F, Yoshikawa K, Uchida T, Fukuda T (2005) In situ formation of a gel microbead for indirect laser micromanipulation of microorganisms. Appl Phys Lett 87(19):191108

14. Ito K, Sakuma S, Yokoyama Y, Arai F (2014) On-chip gel-valve using photoprocessable thermoresponsive gel. ROBOMECH J 1(1):1-8

15. Hayakawa T, Sakuma S, Fukuhara T, Yokoyama Y, Arai F (2014) A single cell extraction chip using vibration-induced whirling flow and a thermoresponsive gel pattern. Micromachines 5(3):681-696

16. d'Eramo L, Chollet B, Leman M, Martwong E, Li M, Geisler H, Dupire J, Kerdraon M, Vergne C, Monti F et al (2018) Microfluidic actuators based on temperature-responsive hydrogels. Microsyst Nanoeng 4(1):1-7

17. Lee W, Kalashnikov N, Mok S, Halaoui R, Kuzmin E, Putnam AJ, Takayama S, Park M, McCaffrey L, Zhao R et al (2019) Dispersible hydrogel force sensors reveal patterns of solid mechanical stress in multicellular spheroid cultures. Nat Commun 10(1):1-14

18. Koike Y, Yokoyama Y, Hayakawa T (2020) Light-driven hydrogel microactuators for on-chip cell manipulations. Front Mech Eng 6:2

19. Wada H, Koike Y, Yokoyama Y, Hayakawa T (2020) Evaluation of the response characteristics of on-chip gel actuators for various single cell manipulations. IEEE Robot Autom Lett 5(4):5205-5212

20. Yokoyama Y, Umezaki M, Kishimura T, Tamiya E, Takamura Y (2011) Microand nano-fabrication of stimulus-responsive polymer using nanoimprint lithography. J Photopolym Sci Technol 24(1):63-70

21. Tompkins N, Fraden S (2016) An inexpensive programmable illumination microscope with active feedback. Am J Phys 84(2):150-158

22. Steyrer B, Busetti B, Harakály G, Liska R, Stampfl J (2018) Hot lithography vs. room temperature dlp $3 d$-printing of a dimethacrylate. Addit Manuf 21:209-214

\section{Publisher's Note}

Springer Nature remains neutral with regard to jurisdictional claims in published maps and institutional affiliations.

\section{Submit your manuscript to a SpringerOpen ${ }^{\odot}$ journal and benefit from:}

- Convenient online submission

- Rigorous peer review

- Open access: articles freely available online

- High visibility within the field

- Retaining the copyright to your article

Submit your next manuscript at $\boldsymbol{\nabla}$ springeropen.com 